\title{
Timing Characterization of Helium-4 Fast Neutron Detector with EJ-309 Organic Liquid Scintillator
}

\author{
Yinong Liang ${ }^{1}$, Ting Zhu, Andreas Enqvist
}

\begin{abstract}
Recently, the Helium-4 gas fast neutron scintillation detectors is being used in time-sensitive measurements, such timeof-flight and multiplicity counting. In this paper, a set of time aligned signals was acquired in a coincidence measurement using the Helium-4 gas detectors and EJ-309 liquid scintillators. The high-speed digitizer system is implanted with a trigger moving average window (MAW) unit combing with its constant fraction discriminator (CFD) feature. It can calculate a "time offset" to the timestamp value to get a higher resolution timestamp (up to $50 \mathrm{ps}$ ), which is better than the digitizer's time resolution (4 ns) [1]. The digitized waveforms were saved to the computer hard drive and post processed with digital analysis code to determine the difference of their arrival times. The full-width at half-maximum (FWHM) of the Gaussian fit was used as to examine the resolution. For the cascade decay of Cobalt-60 (1.17 and 1.33 MeV), the first version of the Helium-4 detector with two Hamamatsu R580 photomultipliers (PMT) installed at either end of the cylindrical gas chamber $(20 \mathrm{~cm}$ in length and $4.4 \mathrm{~cm}$ in diameter) has a time resolution which is about 3.139 ns FWHM. With improved knowledge of the timing performance, the Helium-4 scintillation detectors are excellent for neutron energy spectrometry applications requiring high temporal and energy resolutions.

Index Terms-Helium-4 detectors, time resolution, TOF.
\end{abstract}

\section{INTRODUCTION}

$\mathrm{T}$ ime-of-Flight (TOF) technique is based on a pulsed neutron beam, spanning over a wide range of energies, travels a given distance before reaching the detector under study [2]. In this work, the kinetic energy of the neutrons is determined by calculating the difference between the timestamp reported by the analog-digital-converter (ADC) of each neutron event in the Helium-4 detector and the start time of the generator pulse. With a known distance between the target and the detector, the TOF was converted to determine the incident neutron energy. The outputs of the TOF measurement can be used in many neutron spectrometry applications such as detector light response calibration and mathematical spectrum unfolding [3]. The relative energy resolution of a TOF measurement is shown in (1) [2] as a first approximation. The moderation time in the target-moderator assembly, and the detector response, contribute to the fact that even for neutrons with a given kinetic

${ }^{1}$ Submitted on 6/1/2017. This material is based upon work supported by the Department of Energy under Award Number: DE-NE0008459. energy, the measured TOF could have a distribution.

$$
\frac{\Delta E_{n}}{E_{n}}=2 \times \sqrt{\left(\frac{\Delta t}{t}\right)^{2}+\left(\frac{\Delta L}{L}\right)^{2}}
$$

As from (1), a high-accuracy TOF results can be achieved by the use of long flight paths (large $\mathrm{L}$ and $\mathrm{t}$ ) and the relative energy resolution can be estimate by knowing the time resolution of the detection system. Previous studies [3] were mainly focused on the TOF based detector response characterization and the spectrum unfolding algorithm development. The accuracy of the TOF would affect the construction of the detectors response function and therefore the precision of the unfolding results. Therefore, it is crucial to characterize the timing performance of the Helium-4 detectors.

\section{MATERIALS AND EXPERIMENTAL METHODS}

In this chapter, two versions of the Helium-4 fast neutron detectors were introduced and compared. For the timing measurement, a Struck SIS3316 16 channel digitizer was used. Its MAW unit is able to characterize the detectors time performance at a higher time resolution (less than $4 \mathrm{~ns}$ ) through time interpolation.

\section{A. Helium-4 Gas Fast Neutron Scintillation Detector}

The Helium-4 gas fast neutron scintillation detector is based on neutron elastic scattering of Helium-4 nuclei. The Helium-4 nucleus gains kinetic energy from neutrons, and it will excite or ionize other helium atoms along its path within the detector volume. The gas pressure of Helium-4 is about 150 bar that the penetration distance of recoil is much smaller than the diameter of the gas chamber (see Fig.1). Scintillation photons are then be generated from the de-excitation process of the helium excimers. The first version of the Helium-4 detector has two Hamamatsu R580 photomultiplier tubes (PMT) on the both ends of the gas chamber are set up in coincidence mode so as to suppress the recording of PMT noise signals [4].

The second version has a series of SensL siliconphotomultipliers (SiPM), also known as the Geiger mode avalanche photodiode (GAPD) or solid state photomultiplier (SSPM), immersed inside a much longer $(60 \mathrm{~cm})$ pressurized (180 bars) helium-4 gas chamber [5]. The detector has a unique

Y. Liang is a PhD student in the University of Florida, Gainesville, FL, 32611, USA (email: yinong@ufl.edu). 
design of three optically separated segments in which 12 SiPM pairs (four in each segment) are positioned equilaterally across the detector to allow for them to be fully immersed in the Helium-4 gas volume; consequently, no additional optical interfaces are necessary. A schematic of the SiPM detectors can be seen in Fig. 2.

Typically, PMTs have faster signal rise time and lower dark count rates than SiPMs, but SiPMs have a high gain and quantum efficiency (QE), and are also very compact and rugged, insensitive to magnetic fields, and can be cost efficient when fabricated on a large scale [6]. For SiPM based detectors, they usually present longer tails due to the large output capacitance $(\mathrm{C})$ associated with its relatively large pixel size and the input resistance $(\mathrm{R})$ of the subsequent amplifier [7]. Therefore, the SiPM based detectors are expected having worse time resolution than PMT based ones. Measurements for the SiPM based detectors will be conducted in future work due to their more complex design.

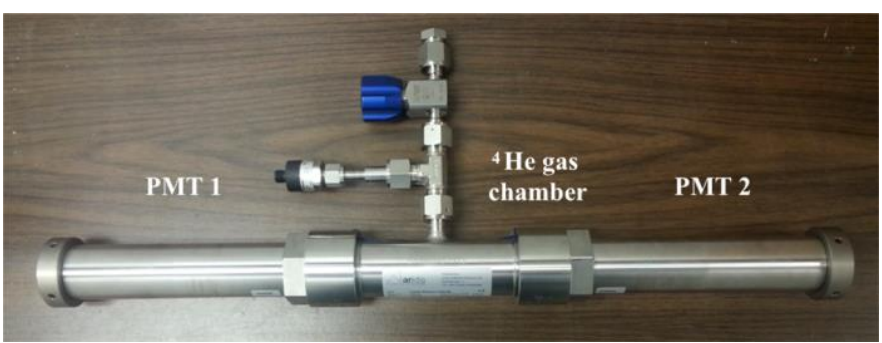

Fig. 1. A PMT based Helium-4 fast neutron detector. The active volume at the center is pressurized to 150 bar.

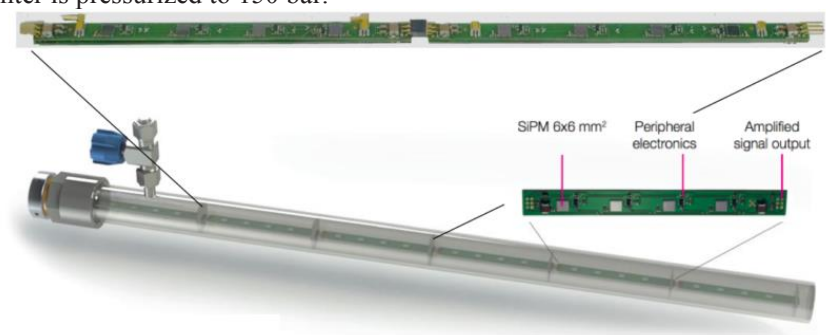

Fig. 2. Schematic of a SiPM based Heliium-4 detector.

The Helium-4 gas fast neutron scintillation detector has been demonstrated to be one of promising candidates to be used in nuclear safeguards and homeland security applications [8]. Energy information of incident neutrons could be retained in measurements based on the Helium- 4 detector because no moderating materials are used. They also have fast response time on the order of nanoseconds and low sensitivity to $\gamma$-rays. In addition, neutrons and $\gamma$-rays can be well separated because of the good pulse shape discrimination capability [4]. These properties make the Helium-4 detector especially attractive for neutron detection in ultra-high $\gamma$-ray background. For example, in spent nuclear fuel assay, the $\gamma$-ray input count rate from the long-lived fission fragments could easily reach as high as $10^{\wedge} 6$ counts per second. The low sensitivity and good PSD performance will be greatly beneficial to the improvement of neutron detection throughput at high-rate.

\section{B. SIS3316 16 channel VME digitizer}

The data acquisition was performed with a Struck SIS3316
16 channel digitizer card with $250 \mathrm{MHz}$ sampling speed and 14bit resolution. It combines high channel density per card with the availability of greatly enhanced FPGA resources per channel and a flexible analog input stage followed by high resolution digitizer/ADC chips. The implemented FPGA firmware of the SIS3316 has many advantages which allow the timing characterization measurement. This section will mainly focus on the digitizer's trigger MAW unit and higher "timestamp" resolution ability.

For the internal trigger generation, a trapezoidal finite impulse response (FIR) filter with CFD feature is implemented for each ADC channel to generate a trigger signal. The initial ADC signal goes in to a MAW at first, and a moving average (MA) will be performed over the programmable peaking time. When the CFD feature is enabled, the trigger logic will be armed if the actual trapezoidal value goes above the programmable threshold value. A trigger pulse would be generated only if the trigger logic is armed and the actual trapezoidal value falls below the half of its maximum value. Three MAW values from FIR trigger trapezoidal can be used to get a "timestamp" which is better than the sampling period. With these three MAW values (maximum value, value after trigger, value before trigger), it is possible to make a linear interpolation between the two values (before and after) to calculate a "negative time offset" to the latched timestamp value to get the "real" timestamp [1].

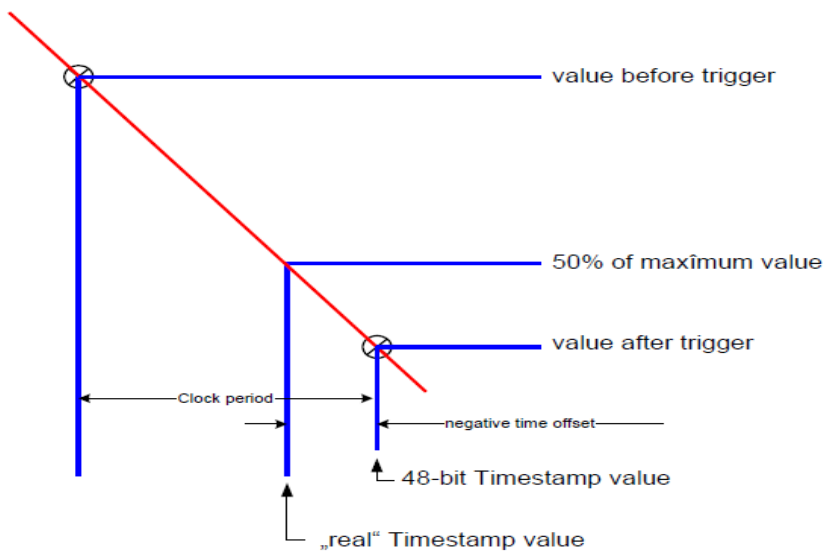

Fig. 3. Linear interpolation by using three MAW values.

\section{Time resolution measurement}

The hydrogen-based organic liquid scintillator EJ-309 (3" diameter x 3" long) is coupled with a 3" diameter ETEL 9821KB PMT. EJ-309 scintillators are widely used in both neutron and gamma detections and can be used as a reference and comparison with Helium-4 detectors. Time resolution of EJ-309 scintillators was measured at first. Two EJ-309 scintillators with same dimensions were located at an equal distance $(5 \mathrm{~cm}$ from the Cobalt- 60 gamma source to the face of the detectors) at opposite sides. Previous research has proved that the two gamma rays are angularly correlated, with a maximum probability at 180 degrees. Therefore the detectors were placed horizontally with respect to the outgoing gamma rays. Both detectors were set up in the time-coincidence mode (coincidence window length as $40 \mathrm{~ns}$ ) such that each detector 
detected one of the two gammas rays emitted from the same Cobalt-60 beta-decay.

The experimental setup for measuring the PMT based Helium-4 detectors was basically the same except for substituting one EJ-309 to one PMT based Helium-4 detector and the employment of a linear fan-in/fan-out units. The anode signal from the two PMTs were connected to a Phillips Scientific 740 quad linear/logic fan-in/fan-out to maintain signal integrity, and was summed into one pulse with twice of the amplitude. The analog output signals were then digitized with a SIS3316-250MHz-14bit ADC chip with a dynamic range of $1.75 \mathrm{~V}$. The programmable trigger threshold was adjust to relatively low $(6.2 \mathrm{mV})$ and the shaping delays $(10 \mathrm{~ns})$ were optimized to obtain the best possible time resolution. The digitized data acquired from Struck SIS3316 was processed offline to obtain the time information of each pulse, and the respective "timestamps" reported by the ADC were compared. One thing to be noticed is the intrinsic time resolution of the analog and digital modules is considered small (115 ps for fanin/fan-out and 65 ps for Struck digitizer) [1], and is negligible when comparing with the nanosecond range of the time resolution for the whole system.

\section{RESULT AND DISCUSSION}

Time resolution of the EJ-309 scintillators and PMT-based Helium-4 detectors were measured and compared in this chapter. For EJ-309 scintillator, the dependence of the threshold versus time resolution was explored. With increasing threshold, it tends to yield better time resolution. For the Helium-4 detectors, pulses from PMTs were analyzed individually and then summed together for comparison.

\section{A. Time resolution calculation}

In a photomultiplier tube, through the two major components inside the tube (photocathode and dynode), the extremely weak light output of a scintillation pulse can be converted to a corresponding usable electrical signal. Because the time required for photoemission in the photocathode or secondary emission from dynodes is very short ( $0.1 \mathrm{~ns}$ or less), the time characteristics of the PMT are determined exclusively by the electron trajectories [9]. The electron transit time of a PMT is defined as the average time difference between the arrival of a photon at the photocathode and the final collection of the subsequent electrons at the anode. In most timing applications, however, the transit time itself is not of the primary interests because if it were always a constant, it would introduce only a fixed delay in the derived signal and can be corrected later in pulse processing. Instead, the spread in transit time is a more important quantity because it determines the time width of the pulse of electrons arriving at the anode of the tube, and can somehow indicate the detection system's time resolution.

Due to various electron trajectories as mentioned above, there will be a slightly difference in the reported "timestamp" for each coincidence gamma rays from the Cobalt-60 cascade decay. The time difference is calculated as:

$$
\Delta T=\text { Timestamp }_{\text {Det } 1}-\text { Timestamp } p_{\text {Det } 2}
$$

The time difference can by fitted by a Gaussian function. For peaks whose shape is Gaussian with standard deviation $\sigma$, the full width at half maximum (FWHM) is given by $2.35 \sigma$.

$$
F W H M=2.35 \sigma
$$

The standard deviation of the timing difference is calculated as below based on error propagation.

$$
\sigma_{\Delta T}^{2}=\sigma_{\Delta \text { Timestamp }}{ }_{\text {Det } 1}^{2}+\sigma_{\Delta \text { Timestamp }}{ }_{\text {Det } 2}^{2}
$$

Combing all the equations above together, the FWHM for each type of detection systems can be obtained.

\section{B. EJ-309 scintillators time resolution}

The experimental setup of EJ-309 scintillator time resolution characterization is as shown in Fig. 4. A Cobalt-60 gamma source was placed at the center of the detector's active volume and was $5 \mathrm{~cm}$ to the face of both detectors. Within 1 minute, 2400 coincidence events were recorded. The detection pulseheight threshold for all measurements is $100 \mathrm{keVee}$.

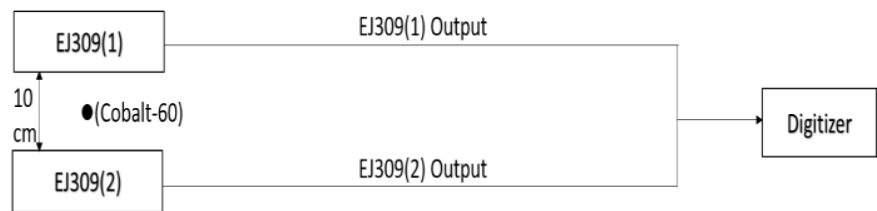

Fig. 4. Experimental setup for EJ-309 scintillator time resolution characterization.

Calibration was conducted as the first step to match the PMT voltages of both detectors. The "MeV-electron-equivalent" conversion was performed as in previous work [10]. Fig. 5 shows the distribution of the difference in the reported "timestamp" for each detector. It is modeled by a Gaussian function at a threshold of $100 \mathrm{keVee}$. The FWHM was calculated as 1.239 ns. From Fig. 6 and Fig. 7, the detector achieves better time resolution as threshold increases at a sacrifice of statistics since more coincidence events were rejected. Thus $100 \mathrm{keVee}$ was chosen and was kept in later analysis.

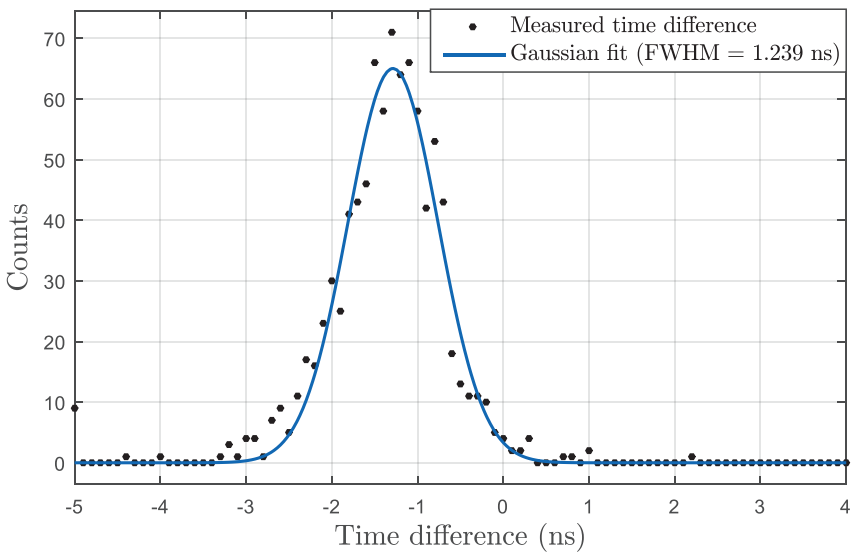

Fig. 5. Timestamp difference distribution of two EJ-309 scintillators as modeled in Gaussian function. 


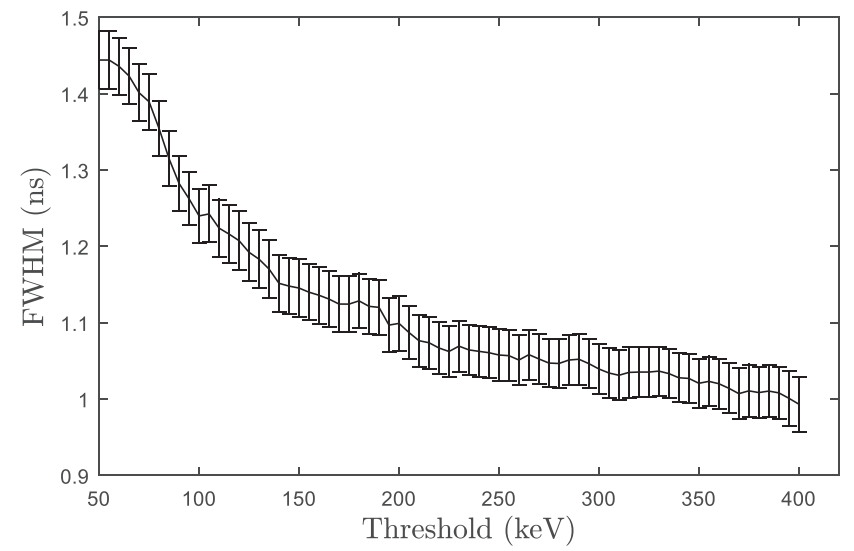

Fig. 6. The FWHM as a function of Threshold.

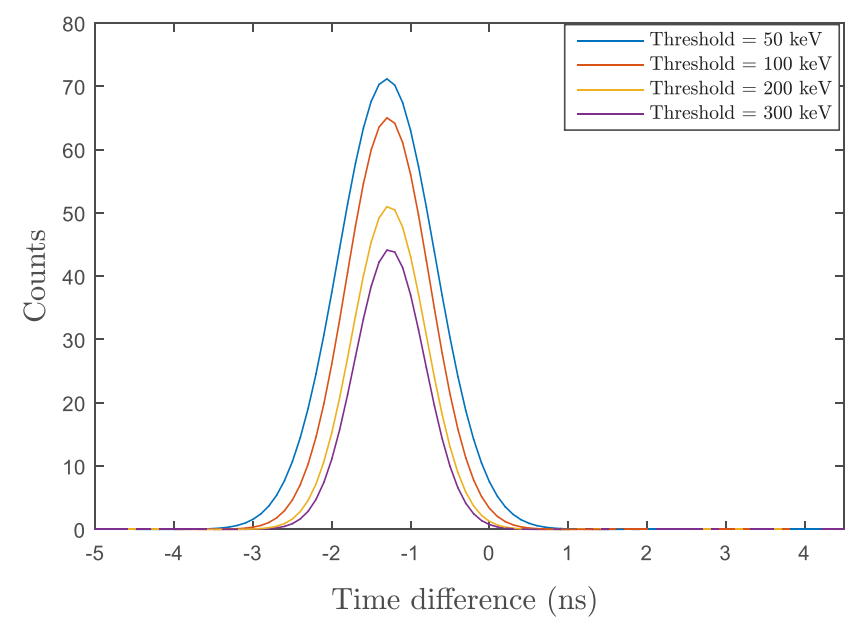

Fig. 7. Gaussian fit for different threshold values.

\section{PMT-based Helium-4 fast neutron detectors}

Fig. 8 shows the experimental setup for measuring the PMTbased Helium-4 fast neutron detectors. The PMT high voltage was set as 1356V (for node A003E) and 1538V (for node A003D) respectively to match the pulse amplitude based on previous calibration [4]. A Cobalt-60 gamma source was placed at the center of the detector's active volume and was $3 \mathrm{~cm}$ to the face of both detectors in order to achieve a higher count rate. Two leads were used as collimator. Only the Gamma rays interacted at the center of the detector were counted, thus the FWHM is independent of the interaction location. In 15 minutes, 5086 coincidence events were recorded. The pulses from two PMTs were summed in the fan-in/fan-out module and then measured on coincident mode with the EJ-309 output signals. From the equations in last section, by knowing the FWHM of EJ-309 scintillators, the FWHM for the summed signals was calculated as 3.139 ns, which was shown in Fig. 9. In addition, time resolution characterization for individual PMT was also conducted, and the time difference was calculated and compared separately with the summed PMT outputs as in Fig. 10. The FWHM of the time spread from the two PMTs are calculated as $2.820 \mathrm{~ns}$ (for node A003E) and $2.851 \mathrm{~ns}$ (for node A003D) respectively. As expected, summing the outputs together brought larger variance thus lead to larger FWHM.

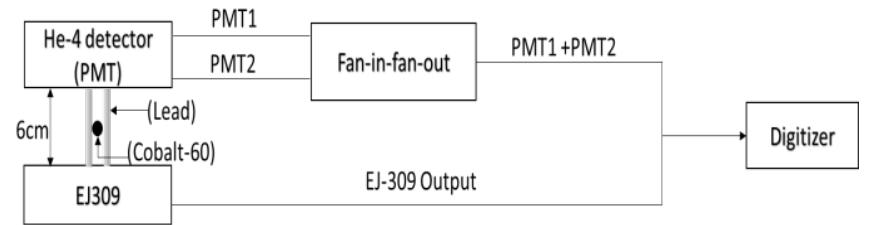

Fig. 8. Experimental setup for the PMT-based Helium-4 fast neutron detectors.

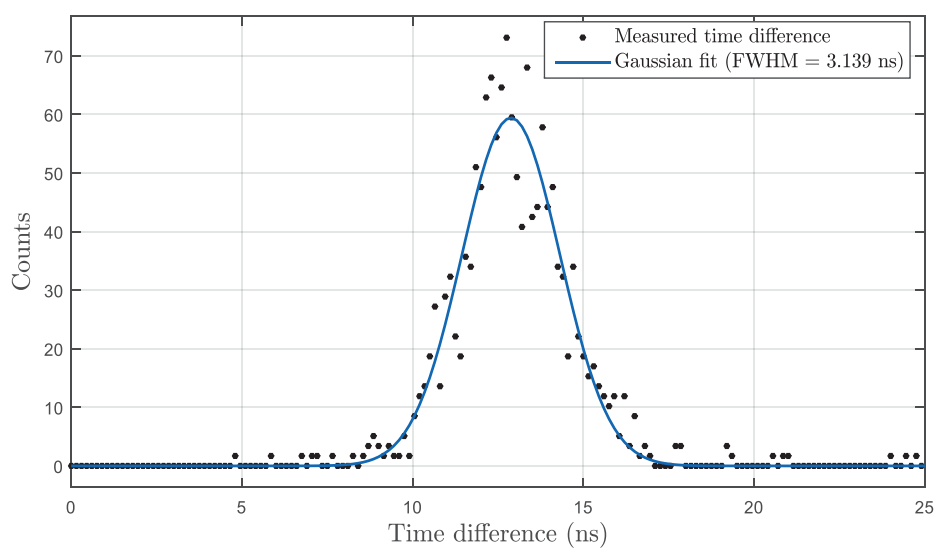

Fig. 9. Timestamp difference (EJ-309 and Helium-4 detectors) distribution as modeled in Gaussian function.

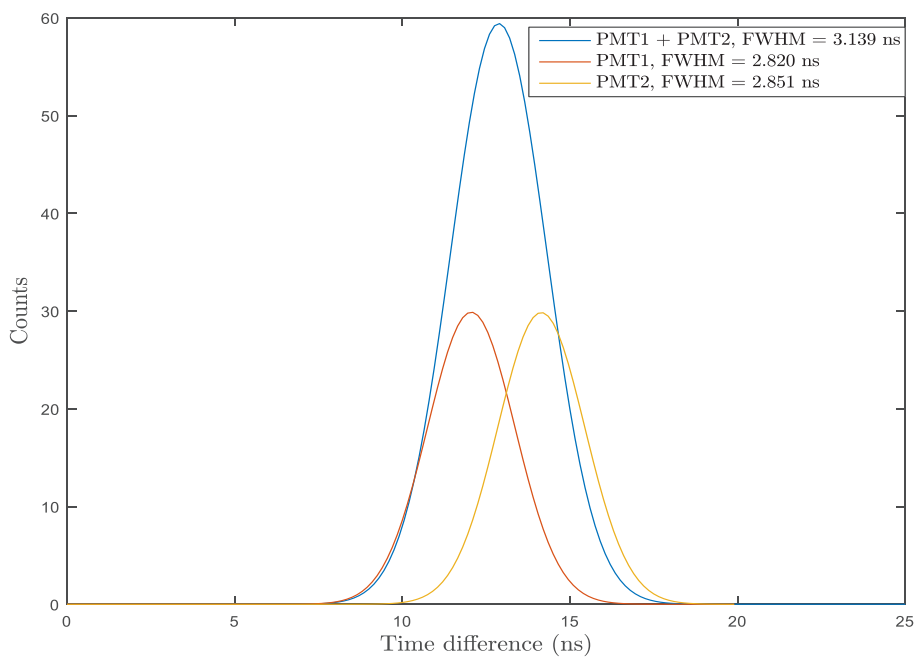

Fig. 10. Gaussian fit for the timestamp difference from summed as well individual PMTs.

By know the time resolution of the Helium-4 detection system, an estimation of the relatively energy resolution of the TOF measurement in previous work [11] can be achieved as in Table 1.

\begin{tabular}{lll}
\hline \hline $\begin{array}{l}\text { TOF } \\
(\mathrm{ns})\end{array}$ & Energy $(\mathrm{MeV})$ & $\Delta \mathrm{E} / \mathrm{E}(\%)$ \\
\hline 295.2 & 6 & 4.25 \\
361.5 & 4 & 3.47 \\
511.1 & 2 & 2.46 \\
723 & 1 & 0.87 \\
\hline \hline
\end{tabular}

Table. 1. Relatively energy resolution of previous TOF measurement (with a distance of $10 \mathrm{~m}$ ) by knowing the Helium-4 detectors time resolution.

\section{CONCLUSIONS}

In this paper, the time resolution of PMT-based Helium-4 fast neutron scintillation detectors was measured and compared 
with a hydrogen-based organic liquid scintillator (EJ-309) for the first time. For the cascade decay of Cobalt-60 (1.17 and $1.33 \mathrm{MeV})$, the Helium-4 detector was measured and yielded a time resolution as $3.139 \mathrm{~ns}$ FWHM. It is slightly worse (2 ns) than EJ-309 scintillators. The time resolution depends mainly on two parameters: the signal rise time and the signal to noise ratio, both of which should be high to give good time resolution [12]. For the Helium-4 detector, its low electron density leads to low gamma interaction probability. Additionally, Gamma rays also have a lower light yield therefore longer scintillation collection time in gaseous helium scintillation when compared to liquid scintillators. Thus, the gamma interaction based characterization measurement could compromise the Helium-4 detector's time resolution. Helium-4 detectors are designed for neutron detection with good gamma rejection and energy discrimination ability. The measured time resolution provides a reference to correct the uncertainties associated with TOF measurement and can be used to examine the detector's energy resolution in later work. Future work will also include the effect of source position, SiPM-based time resolution characterization, PMT operation voltage, and the effects of various digital analysis techniques (leading-edge discrimination [13], constant-fraction-zero-crossing [13], and digital-constant-fraction discrimination [14]) on the measured time resolution."

\section{REFERENCES}

[1] "16 Channel VME Digitizer User Manual," 2015.

[2] C. Guerrero et al., "Performance of the neutron time-of-flight facility n_TOF at CERN,” Eur. Phys. J. A, vol. 49, no. 2, pp. 1-15, 2013.

[3]R. P. Kelley et al., "Neutron response function characterization of $4 \mathrm{He}$ scintillation detectors," Nucl. Instruments Methods Phys. Res. Sect. A Accel. Spectrometers, Detect. Assoc. Equip., vol. 793, pp. 101-107, 2015.

[4]T. Zhu et al., "Improved fission neutron energy discrimination with $4 \mathrm{He}$ detectors through pulse filtering," Nucl. Instruments Methods Phys. Res. Sect. A Accel. Spectrometers, Detect. Assoc. Equip., vol. 848, September 2016, pp. 137-143, 2017.

[5]R. P. Kelley, T. Zhu, S. Gokhale, Y. Liang, H. Chung, S. Kiff, D. Murer, H. Ray, J. E. Baciak, "Neutron Measurements with Extended Range Helium-4 Detectors in High Gamma Environments," in 2016 IEEE Symposium on Radiation Measurements and Applications (SORMA West), 2016.

[6]J. Y. Yeom, R. Vinke, and C. S. Levin, "Optimizing timing performance of silicon photomultiplier based scintillation detectors," IEEE Nucl. Sci. Symp. Conf. Rec., pp. 3119-3121, 2012.

[7]S. Dolinsky, G. Fu, and A. Ivan, "Timing resolution performance comparison for fast and standard outputs of SensL SiPM," IEEE Nucl. Sci. Symp. Conf. Rec., 2013.

[8]H. Chung, R. P. Kelley, W. Lee, Y. H. Chung, and K. A. Jordan, "Spent nuclear fuel cask and storage monitoring with Helium-4 scintillation fast neutron detectors," Proc. Korean Nucl. Soc. 2014 Fall Meeting, 2014.

[9] G. F. Knoll, Radiation detection and measurement. Don Fowley, 2010.

[10]H. Wang, D. Carter, T. N. Massey, and A. Enqvist, "Neutron light output function and resolution investigation of the deuterated organic liquid scintillator EJ-315," Radiat. Meas., vol. 89, pp. 99-106, 2016.

[11]Y. Liang et al., "Neutron spectrum unfolding with $4 \mathrm{He}$ fast neutron detectors using an iterative least squares approach." unpublished.

[12]A. Ronzhin et al., "Waveform digitization for high resolution timing detectors with silicon photomultipliers," Nucl. Instruments Methods Phys. Res. Sect. A Accel. Spectrometers, Detect. Assoc. Equip., vol. 668, pp. 94-97, 2012.
[13]J. Nissilä, K. Rytsölä, R. Aavikko, A. Laakso, K. Saarinen, and P. Hautojärvi, "Performance analysis of a digital positron lifetime spectrometer," Nucl. Instruments Methods Phys. Res. Sect. A Accel. Spectrometers, Detect. Assoc. Equip., vol. 538, no. 1-3, pp. 778-789, 2005.

[14]D. G. Cussans and H. F. Heath, "Optimization of the pulse arrival time determination in the ZEUS central tracking detector FADC system," Nucl. Inst. Methods Phys. Res. A, vol. 362, no. 2-3, pp. 277-282, 1995. 